\title{
Prevalence, risk factors, and complications associated with hyponatraemia following elective primary hip and knee arthroplasty
}

\author{
Emma Cunningham ${ }^{1}$, Nicola Gallagher ${ }^{2^{*}}$ D, Paul Hamilton ${ }^{3,4}$, Leeann Bryce ${ }^{2}$ and David Beverland ${ }^{2}$
}

\begin{abstract}
Background: Hyponatraemia, defined as a serum sodium [Na] concentration below $135 \mathrm{mmol} / \mathrm{L}$, is common following surgery. As inpatient peri-operative stays shorten, there is a need to recognise pre-operative risk factors for post-operative hyponatraemia and complications associated with a peri-operative drop in Na. This audit aimed to investigate the prevalence of, risk factors for, and complications associated with hyponatraemia following elective primary hip and knee arthroplasty.

Methods: Data were collected within a retrospective audit of inpatient complications and unplanned reattendance or readmission at hospital in consecutive elective primary hip and knee arthroplasty patients in a single high throughput elective primary joint unit. The hospital's electronic database identified 1000 patients who were admitted electively between February 2012 and June 2013 under the care of a single consultant orthopaedic surgeon for either total hip arthroplasty, total knee arthroplasty, or uni-compartmental knee arthroplasty. Groups were compared using appropriate tests, including chi-square analysis (or Fisher's exact test), Mann-Whitney U test, Kruskal-Wallis test, and Wilcoxin signed-rank test. Logistic regression analysis was used to determine factors associated with hyponatraemia.
\end{abstract}

Results: Of the total 1000 patients, 217 (21.7\%) developed post-operative hyponatraemia. Of these, 177 (81.6\%) had mild ( $\mathrm{Na}$ 130-134 mmol/L), 37 (17.1\%) had moderate ( $\mathrm{Na} \mathrm{125-129} \mathrm{mmol/L),} \mathrm{and} 3(1.4 \%)$ had severe ( $\mathrm{Na}<125 \mathrm{mmol} / \mathrm{L})$ hyponatraemia. In multivariate analysis, age, pre-operative $\mathrm{Na}$, and fasting glucose on day 1 remained significantly associated with having hyponatraemia post-operatively. There were no significant differences in reattendance at emergency departments and/or readmission within 90 days between those who had post-operative hyponatraemia whilst in hospital $(39 / 217=18.0 \%)$ and those who did not $(103 / 783=13.2 \%)$, or between those who were discharged with hyponatraemia $(18 / 108=16.7 \%)$ and those discharged with normal $\mathrm{Na}(124 / 880=14.1 \%)$.

Conclusion: Approximately one fifth of elective joint arthroplasty patients had post-operative hyponatraemia. In these patients, older age, lower pre-operative $\mathrm{Na}$ and higher fasting glucose predicted post-operative hyponatraemia. We found no evidence that those discharged with hyponatraemia had more reattendance at emergency departments or readmission to hospital. We suggest that otherwise well patients with mild hyponatraemia can safely be discharged and followed up in the community.

Keywords: Hyponatraemia, Sodium, Prevalence, Risk factors, Total hip arthroplasty, Total knee arthroplasty, Unicompartmental knee arthroplasty

\footnotetext{
* Correspondence: Nicola.Gallagher@belfasttrust.hscni.net

${ }^{2}$ Outcomes Unit, Musgrave Park Hospital, Belfast Health and Social Care Trust, Stockman's Lane, Belfast BT9 7JB, Northern Ireland

Full list of author information is available at the end of the article
}

\section{$\triangle B M C$}

(c) The Author(s). 2021 Open Access This article is licensed under a Creative Commons Attribution 4.0 International License, which permits use, sharing, adaptation, distribution and reproduction in any medium or format, as long as you give appropriate credit to the original author(s) and the source, provide a link to the Creative Commons licence, and indicate if changes were made. The images or other third party material in this article are included in the article's Creative Commons licence, unless indicated otherwise in a credit line to the material. If material is not included in the article's Creative Commons licence and your intended use is not permitted by statutory regulation or exceeds the permitted use, you will need to obtain permission directly from the copyright holder. To view a copy of this licence, visit http://creativecommons.org/licenses/by/4.0/ The Creative Commons Public Domain Dedication waiver (http://creativecommons.org/publicdomain/zero/1.0/) applies to the data made available in this article, unless otherwise stated in a credit line to the data. 


\section{Introduction}

Hyponatraemia, defined here as a serum sodium $[\mathrm{Na}]$ concentration below $135 \mathrm{mmol} / \mathrm{L}$, is common following surgery (Spasovski et al., 2014; Rudge et al., 2014). In vivo regulation of $\mathrm{Na}$ and water is complex, and surgery may lead to a reduced $\mathrm{Na}$ concentration via several pathways. These likely include blood loss leading to stimulation of baroreceptors and appropriate production of antidiuretic hormone $(\mathrm{ADH})$, the inflammation cascade (Park and Shin, 2013), pain, vomiting, fever, positive pressure ventilation, anaesthesia, and analgesia (Hennrikus et al., 2015; Upadhyay et al., 2006; Biswas and Davies, 2007). Many of the latter are linked with so-called 'inappropriate' ADH release, accounting for around one third of hyponatraemia cases. Hyponatraemia is caused by an excess of total body water when compared to total body sodium content; therefore, volume status and osmolality play a key role (Rondon and Badireddy, 2021).

Whilst a small, transient, post-operative drop in Na may be normal and appropriate (estimates range from 0.2 to 3 mmol, with duration less than $48 \mathrm{~h}$ ) (Hennrikus et al., 2015; Harris et al., 2015), a significant hyponatraemia may be accompanied with cerebral oedema and associated morbidity and mortality. Hyponatraemia may also indicate underlying physiological vulnerabilities, being associated as it is, with increasing age, comorbidity, and polypharmacy (Udy et al., 2005; Hawkins et al., 2003; Mohan et al., 2013; Bissram et al., 2007). Similarly, it is not clear whether the associations with increased length of stay (LOS) and mortality after surgery (Hennrikus et al., 2015; Nagler et al., 2014; Madsen et al., 2016; Tinning et al., 2015) are due to the effects of hyponatraemia or to associated issues. For example, Leung et al. (2012) have shown that low pre-operative $\mathrm{Na}$ is associated with negative outcomes after surgery, suggesting that both the hyponatraemia itself and the negative outcomes may reflect underlying problems (Leung et al., 2012).

Existing studies of post-operative hyponatraemia have tended to encompass different surgical groups, for example, combining elective and emergency populations (Hennrikus et al., 2015; Harris et al., 2015; McCausland et al., 2014; Cecconi et al., 2016), with very few concentrating on elective arthroplasty populations (Abola et al., 2019; Sah, 2014; Guglielminotti et al., 2003).

Current surgical practices are less likely to induce hyponatraemia (e.g. surgeries are shorter and blood loss lower) whilst regular laboratory testing and improved clinical awareness are such that post-operative hyponatraemia is more likely to be identified than previously. At the same time, we have two potentially conflicting trends in perioperative medical care. Firstly, older and comorbid patients are presenting for elective orthopaedic surgery. Secondly, we are moving towards shorter inpatient stays and even day case arthroplasty. This means we need to better identify those with underlying vulnerabilities who are at risk of hyponatraemia and other negative outcomes, whilst minimising unnecessary tests for those likely to tolerate a small and short-lived drop in Na concentration. Regular electrolyte testing reduces the risk of missing hyponatraemia; however, Kildow et al. (2018), have suggested that many such tests may be unnecessary (Kildow et al., 2018). Whilst thorough guidelines exist regarding identification and management of hyponatraemia which all reference, and are relevant to, post-operative hyponatraemia, specific guidance covering this area is lacking. The American guidelines report post-operative hyponatraemia as a syndrome of inappropriate antidiuretic hormone secretion (SIADH) and estimate a duration of 2-3 days (Verbalis et al., 2013).

There is currently a lack of evidence and guidance regarding the management of post-operative hyponatraemia for enhanced recovery programmes following arthroplasty. We have succeeded in reducing LOS whilst maintaining a high quality of evidence-based care. We aim to improve our service further, but this requires explicit understanding of the challenges we face. Implant survivorship is well reported in the United Kingdom (UK) (NJR, 2013; NJR, 2018). Medical complications have been investigated less often, a notable exception being the Scottish Arthroplasty Project, which records a small number of specific outcomes.

The aims of this audit were to establish (i) the incidence of and risk factors associated with hyponatraemia following elective primary total hip arthroplasty (THA), total knee arthroplasty (TKA), and uni-knee arthroplasty (UKA); (ii) the associations between post-operative hyponatraemia and other post-operative complications; and (iii) the natural history/timing of post-operative hyponatraemia in our population.

\section{Methods}

Data were collected within a retrospective audit of inpatient complications and unplanned reattendance and readmission at hospital in consecutive elective primary hip and knee arthroplasty patients in a high throughput elective primary joint unit with the goal of improving patient care. The project was registered with the relevant Trust's Standards, Quality and Audit Department. The hospital's electronic database was used to identify 1000 patients who were admitted electively beginning 29th February 2012 (end date 25th June 2013) under the care of a single consultant orthopaedic surgeon for either THA, TKA, or UKA to a single surgical unit. At this time, spinal anaesthesia was given to most patients, and TKA and UKA patients routinely received a femoral nerve block. IV fluid was at the discretion of the anaesthetist. Routine discharge was on day 2 for THA and UKA patients and day 3 for TKA patients. A total of 
1000 patients was arbitrarily chosen in an attempt to attain a representative sample size.

\section{Standard Surgical Technique and Peri-operative Practice}

All THAs were performed in the lateral decubitus position using the posterior approach with a cementless CorailPinnacle combination (DePuy Synthes, Leeds UK). All TKAs were performed using a cementless low contact stress (LCS) rotating platform prosthesis without patellar resurfacing (DePuy Synthes, Leeds, UK). All UKA procedures were performed using the medial cementless Oxford uni-compartmental implant (Biomet, Warsaw, IN). Venous thromboembolism and antimicrobial prophylaxis were administered according to local policy at the time (Ogonda et al., 2016). Of note, antibiotic prophylaxis consisted of $2 \mathrm{~g}$ of flucloxacillin and $1.5 \mathrm{mg} / \mathrm{kg}$ of gentamicin given intravenously prior to knife to skin, followed by three further intravenous doses of $2 \mathrm{~g}$ of flucloxacillin at 6 , 12 , and $18 \mathrm{~h}$ post-operatively. Patients with a history of penicillin allergy or methicillin-resistant Staphylococcus aureus (MRSA) received $10 \mathrm{mg} / \mathrm{kg}$ teicoplanin and 1.5 $\mathrm{mg} / \mathrm{kg}$ gentamicin intravenously once only prior to knife to skin. Tranexamic acid was administered to patients deemed at high risk of bleeding, including those with rapidly progressive osteoarthritis.

Routine practice during these time periods included routine checking of electrolytes on day 1 and day 2 . Post-operative hyponatraemia was investigated and managed by the attending medical team. In Northern Ireland, medical staff are advised to manage hyponatraemia along standard lines using published guidelines (GAIN, 2010).

\section{Data Collection}

Data were collected manually from individual clinical (medical and nursing) notes and local and regional electronic databases by trained staff, including nursing and medical staff. Data collected included baseline demographics, surgical and anaesthetic details, laboratory data, inpatient radiological imaging, inpatient postoperative complications (defined as an event requiring intervention by healthcare staff), LOS (based on date of admission and date of discharge), reattendance at emergency departments (ED), and/or readmission within 90 days and 90-day mortality.

Blood results were taken from the trust's electronic laboratory system. This included surgery blood loss and blood loss at 24 and 48 hours post-operation. Preoperative $\mathrm{Na}$ on the day of surgery was not available in all patients. When available, pre-operative $\mathrm{Na}$, which was measured on the day of surgery, was taken. If this was not available, a pre-operative assessment $\mathrm{Na}$ was taken. Pre-operative assessment varied between 1 and 552 days before surgery, median 97 (interquartile range,
IQR 65.3-141.0). Hyponatraemia was defined as a $\mathrm{Na}<$ $135 \mathrm{mmol} / \mathrm{L}$. This was further categorised as mild, $\mathrm{Na}$ 130-134 mmol/L; moderate, Na 125-129 mmol/L; or severe, $\mathrm{Na}<125 \mathrm{mmol} / \mathrm{L}$ (Spasovski et al., 2014). Of note, fasting glucose was checked for in a subset of patients only. This tended to be at physician request, but it is likely diabetics were overrepresented in the patients who had a fasting glucose checked.

\section{Statistical Analysis}

All statistical analyses were carried out using SPSS for Windows (IBM, Version 22.0, Armonk, USA) and all data were assessed for normality using the Shapiro-Wilk test. Descriptive statistics, including absolute number (\%) and median (IQR), were used as necessary. Chi-square analysis (or Fisher's Exact test as appropriate) and Mann-Whitney $\mathrm{U}$ test were used to compare categorical and continuous variables, respectively, between those with and without post-operative hyponatraemia. Kruskal-Wallis testing was used to compare Na between THA, TKA, and UKA patients, since previous studies have found varying susceptibility to hyponatraemia by type of arthroplasty (Hennrikus et al. 2015, Sah, 2014). The Wilcoxin signed-rank test was used to compare pre- and post-operative $\mathrm{Na}$. Logistic regression analysis was used to determine factors associated with hyponatraemia. Variables which displayed a p $<0.1$ in univariate analysis were added to the multivariate logistic regression model. Otherwise, statistical significance was set at the $\mathrm{p}<0.05$ level.

\section{Results}

A total of 1015 patients underwent elective primary hip or knee arthroplasty during the audit period. Of these, one underwent non-arthroplasty surgery, one was a duplicate record, and notes were not available for $13 \mathrm{pa}-$ tients, leaving 1000 patients. Patients undergoing more than one surgery during the audit period were considered as different audit entries. Thirty-one patients had two primary arthroplasties during the audit period. No patient had more than two surgeries. Of the 1000 joint replacements, 493 (49.3\%) were primary THA, 425 $(42.5 \%)$ were primary TKA and $82(8.2 \%)$ were primary UKA. One of the most common post-operative complications identified was hyponatraemia.

\section{Incidence and predictors of post-operative hyponatraemia}

Out of the total 1000 patients, 217 (21.7\%) had postoperative hyponatraemia, i.e. $\mathrm{Na}<135 \mathrm{mmol} / \mathrm{L}$. Of these, $177(81.6 \%)$ had mild (Na 130-134 mmol/L), 37 (17.1\%) had moderate ( $\mathrm{Na} 125-129 \mathrm{mmol} / \mathrm{L})$, and 3 (1.4\%) had severe $(\mathrm{Na}<125 \mathrm{mmol} / \mathrm{L})$ hyponatraemia. Three patients had post-operative hypernatraemia $(\mathrm{Na}>$ 
$145 \mathrm{mmol} / \mathrm{L}$ ). For the purposes of the analyses, these patients were included in the non-hyponatraemia group.

Baseline characteristics and peri-operative variables for all 1000 patients are shown in Table 1 with a comparison of those with and without post-operative hyponatraemia. Of the $42(4.2 \%)$ patients who had hyponatraemia pre-operatively (5 moderate and 37 mild), 31 (73.8\%) also had post-operative hyponatraemia, of whom 3 had moderate pre-operative hyponatraemia which remained moderate, 27 had mild pre-operative hyponatraemia which remained mild, and one had mild pre-operative hyponatraemia which became severe. The remaining 11 (26.2\%) who presented with hyponatraemia before surgery had a post-operative $\mathrm{Na}$ of $\geq 135 \mathrm{mmol} / \mathrm{L}$ ( 2 moderate and 9 mild, pre-operatively). The full list of medical complications by incidence in the hyponatraemia group and non-hyponatraemia group is provided in Table 2. Medication use for all 1000 patients and a comparison of those with and without post-operative hyponatraemia are shown in Table 3.

After adjusting for the factors which showed a significance of $\mathrm{p}<0.1$ in the univariate analysis, age, preoperative $\mathrm{Na}$ and fasting glucose on day 1 remained significantly associated with having hyponatraemia postoperation (Table 4). Pre-operative $\mathrm{Na}(\mathrm{mmol} / \mathrm{L})$ was the most statistically significant predictor. Both preoperative $\mathrm{Na}$ and fasting glucose were treated as continuous variables. We found a reduced risk of postoperative hyponatraemia $(\mathrm{OR}=0.66 ; 95 \% \mathrm{CI}=0.55-$ 0.78 ) for every $1 \mathrm{mmol} / \mathrm{L}$ increase in pre-operative $\mathrm{Na}$ and an increased risk of post-operative hyponatraemia $(\mathrm{OR}=1.33$; $95 \% \mathrm{CI}=1.08-1.64)$ for every $1 \mathrm{mmol} / \mathrm{L}$ increase in fasting glucose on day 1 (Table 4).

\section{Associations between post-operative hyponatraemia and other post-operative complications}

Post-operative hyponatraemia was associated with several other post-operative complications in our population. Cardiac arrhythmia, pulmonary oedema, lower respiratory tract infection, pulmonary embolism, confusion, acute kidney injury, and blood transfusion were all more common in those with post-operative hyponatraemia (Table 2). These post-operative complications were also more common in those with post-operative mild hyponatraemia compared to those without postoperative hyponatraemia (results not shown). Further consideration was given to those clinical outcomes associated with hyponatraemia in the literature. Post hoc analysis was completed to determine whether hyponatraemia could be associated with confusion and falls. The number of patients experiencing confusion postoperatively was significantly higher $(p=0.015)$ in those with hyponatraemia $(22 / 217=10.1 \%)$ compared to those without post-operative hyponatraemia $(43 / 779=5.5 \%)$.
This association did not withstand multivariate analysis (Supplementary Table 1). There was no significant difference $(p=0.340)$ in falls between those with hyponatraemia $(5 / 217=2.3 \%)$ compared to those without postoperative hyponatraemia $(10 / 779=1.28 \%)$. There was also no significant difference $(p=0.798)$ in vasovagal episodes between those with hyponatraemia $(50 / 217=$ $23.0 \%)$ compared to those without post-operative hyponatraemia $(186 / 779=23.9 \%)$. Vasovagal episodes included any instance where light-headedness on standing or mobilising necessitated limitations to mobilisation and/or return to bed, as well as the less common episodes of loss of consciousness.

A significantly higher proportion $(\mathrm{p}<0.001)$ of those with hyponatraemia $(138 / 217=63.6 \%)$ had a delayed discharge (LOS over 3 days) compared to those without hyponatraemia $(300 / 779=38.5 \%)$. However, in multivariate analysis, after adjustment for age, pre-operative $\mathrm{Na}$, post-operative $\mathrm{Na}$, ASA grade, type of surgery, transfusion, post-operative confusion, arrhythmia, pulmonary oedema, lower respiratory tract infection, pulmonary embolism, and acute kidney injury, post-operative $\mathrm{Na}$ was no longer associated with delayed discharge (Supplementary Table 2).

There were no significant differences $(p=0.072)$ in reattendance at ED, and/or readmission within 90 days between those who had post-operative hyponatraemia $(39 / 217=18.0 \%)$ and those who did not $(103 / 783=$ $13.2 \%)$. There were also no significant differences ( $p=$ 0.471 ) in reattendance at ED, and/or readmission within 90 days between those who were discharged with hyponatraemia $(18 / 108=16.7 \%)$ and those discharged with normal $\mathrm{Na}(124 / 880=14.1 \%)$. Reasons for reattendance at ED, and/or readmission within 90 days in those with and without post-operative hyponatraemia are provided in Supplementary Table 3. No patients died within 90 days.

\section{The natural history/timing of post-operative hyponatraemia in our population}

Table 5 provides the $\mathrm{Na}$ concentrations for the total 1000 patients with a comparison by arthroplasty type and with and without post-operative hyponatraemia. Of the 108 patients discharged with $\mathrm{Na}<135 \mathrm{mmol} / \mathrm{L}$, 99 patients had mild hyponatraemia, eight patients had moderate hyponatraemia, and one patient had severe hyponatraemia.

Figures 1, 2, and 3 show the mean $\mathrm{Na}$ at preoperation, day 1 , day 2 , and last $\mathrm{Na}$ prior to discharge (Fig. 1, total group and comparison of those with and without post-operative hyponatraemia; Fig. 2, total group and comparison of arthroplasty type; and Fig. 3, total group and comparison of ASA grade). 
Table 1 Pre-operative patient characteristics for total group and comparison of those with and without post-operative hyponatraemia

\begin{tabular}{|c|c|c|c|c|}
\hline & $\begin{array}{l}\text { Hyponatraemia }(\mathrm{Na}<135 \mathrm{mmol} / \mathrm{L}) \\
\text { post-operation }(n=217)\end{array}$ & $\begin{array}{l}\text { No hyponatraemia }(\mathrm{Na} \geq 135 \mathrm{mmol} / \mathrm{L}) \\
\text { post-operation }(n=783)\end{array}$ & $p$-value ${ }^{1}$ & $\begin{array}{l}\text { Total group } \\
(n=1000)\end{array}$ \\
\hline \multicolumn{5}{|l|}{ Gender $(n=1000)$} \\
\hline Females & $135(62.2 \%)$ & $453(57.9 \%)$ & 0.248 & $588(58.8 \%)$ \\
\hline Males & $82(37.8 \%)$ & $330(42.1 \%)$ & & $412(41.2 \%)$ \\
\hline Age (years) $(n=1000)$ & $73.0(67.0-78.0)$ & $68.0(61.0-75.0)$ & $<0.001$ & $69.0(62.0-76.0)$ \\
\hline Height $(\mathrm{cm})(n=754)$ & $162.2(155.7-169.5)$ & $165.0(158.5-173.0)$ & 0.001 & $164.4(158.0-172.5)$ \\
\hline Weight $(\mathrm{kg})(n=996)$ & $80.0(67.6-92.0)$ & $83.0(72.0-95.4)$ & 0.035 & $82.6(71.0-94.1)$ \\
\hline BMI $\left(\mathrm{kg} / \mathrm{m}^{2}\right)(n=991)$ & $29.8(26.3-34.0)$ & $29.9(26.7-33.7)$ & 0.657 & $29.9(26.6-22.8)$ \\
\hline \multicolumn{5}{|l|}{ ASA grade $(n=1000)$} \\
\hline 1 & $7(3.2 \%)$ & $54(6.9 \%)$ & 0.013 & $61(6.1 \%)$ \\
\hline 2 & $161(74.2 \%)$ & $595(76.0 \%)$ & & $756(75.6 \%)$ \\
\hline $3 / 4$ & $49(22.6 \%)$ & $134(17.5 \%)$ & & $183(18.3 \%)$ \\
\hline \multicolumn{5}{|l|}{ Type of arthroplasty $(n=1000)$} \\
\hline Hip & $100(46.1 \%)$ & $393(50.2 \%)$ & 0.391 & $493(49.3 \%)$ \\
\hline Knee & $101(46.5 \%)$ & $324(41.4 \%)$ & & $425(42.5 \%)$ \\
\hline Uni-knee & $16(7.4 \%)$ & $66(8.4 \%)$ & & $82(8.2 \%)$ \\
\hline Length of stay $(n=1000)$ & $5.0(3.0-7.0)$ & $3.0(2.0-4.0)$ & $<0.001$ & $3.0(2.0-5.0)$ \\
\hline $\begin{array}{l}\text { Pre-operative } \mathrm{Na}(\mathrm{mmol} / \mathrm{L}) \\
(n=999)\end{array}$ & $138.0(136.0-140.0)$ & $140.0(138.0-141.0)$ & $<0.001$ & $139.0(138.0-141.0)$ \\
\hline $\begin{array}{l}\text { Blood loss during surgery (ml) } \\
(n=481)^{+}\end{array}$ & $150.0(100.0-212.5)$ & $150.0(100.0-250.0)$ & 0.614 & $150.0(100.0-243.0)$ \\
\hline 24-h blood loss (ml) $(n=989)$ & $1133.7(869.1-1515.2)$ & $1109.0(798.5-1402.4)$ & 0.128 & $1113.7(810.8-1428.1$ \\
\hline 48-h blood loss $(\mathrm{ml})(n=989)$ & 1390.3 (989.0-1817.9) & $1264.8(885.0-1633.9)$ & 0.003 & $1287.7(899.9-1675.8$ \\
\hline $\begin{array}{l}\text { Duration of operation }(\mathrm{min}) \\
(n=1000)\end{array}$ & $53(46-64)$ & $55(47-63)$ & 0.265 & $54(47-63)$ \\
\hline General anaesthetic $(n=1000)$ & $18(8.3 \%)$ & $68(8.7 \%)$ & 0.856 & $86(8.6 \%)$ \\
\hline Spinal block $(n=1000)$ & $212(97.7 \%)$ & $769(98.2 \%)$ & 0.580 & $981(98.1 \%)$ \\
\hline $\begin{array}{l}\text { Peripheral nerve block } \\
(n=1000)\end{array}$ & $142(65.4 \%)$ & $464(59.3 \%)$ & 0.099 & $606(60.6 \%)$ \\
\hline $\begin{array}{l}\text { Transfusion post-operation } \\
(n=961)\end{array}$ & $44(20.3 \%)$ & $50(6.4 \%)$ & $<0.001$ & $94(9.4 \%)$ \\
\hline $\begin{array}{l}\text { Total intravenous fluids on } \\
\text { day of surgery }(\mathrm{ml})(n=392)\end{array}$ & $2500(2210-3000)$ & $2500(2110-3000)$ & 0.565 & $2500(2140-3000)$ \\
\hline $\begin{array}{l}\text { Pre-operative haemoglobin } \\
(\mathrm{g} / \mathrm{L}) \\
(n=894)\end{array}$ & $131.0(121.0-141.0)$ & $136(125.0-145.0)$ & 0.001 & $134.0(124.0-144.0)$ \\
\hline $\begin{array}{l}\text { Fasting glucose (mmol/L) } \\
(n=422)\end{array}$ & $7.4(6.4-8.6)$ & $6.5(5.9-7.6)$ & $<0.001$ & $6.7(5.9-7.8)$ \\
\hline
\end{tabular}

Median (IQR) used for continuous variables and $\mathrm{n}(\%)$ used for categorical variables

Chi-square analysis used to compare categorical variables and Mann-Whitney $U$ test used to compare continuous variables

${ }^{1} p$-value comparing those with post-operative hyponatraemia ( $\mathrm{Na}<135 \mathrm{mmol} / \mathrm{L}$ ) and those with normal sodium values (Na $\left.\geq 135 \mathrm{mmol} / \mathrm{L}\right)$ post-operatively

${ }^{+}$Blood loss during surgery was only available in hip replacement patients

For pre-operative $\mathrm{Na}$, when available, $\mathrm{Na}$ on the day of surgery was taken, if this was not available, pre-assessment $\mathrm{Na}$ was taken

\section{Discussion}

\section{Main findings}

We have found (i) significant associations between age, pre-operative $\mathrm{Na}$ concentration, fasting glucose on day 1, and post-operative hyponatraemia; (ii) that post-operative hyponatraemia is associated with other post-operative complications; (iii) no difference between reattendance at ED and/or readmission rates in those who developed or were discharged with postoperative hyponatraemia; and (iv) people developing post-operative hyponatraemia started from a lower pre-operative $\mathrm{Na}$, and had a bigger drop in $\mathrm{Na}$, than 
Table 2 Number of patients experiencing inpatient events following elective primary hip and knee arthroplasty

\begin{tabular}{|c|c|c|c|c|}
\hline Post-operative inpatient events & $\begin{array}{l}\text { Incidence total } \\
\text { cohort }(n=996)\end{array}$ & $\begin{array}{l}\text { Incidence hyponatraemia group }(\mathrm{Na}<135 \\
\mathrm{mmol} / \mathrm{L}) \text { post-operation }(n=217)\end{array}$ & $\begin{array}{l}\text { Incidence No hyponatraemia group ( } \mathrm{Na} \geq \\
135 \mathrm{mmol} / \mathrm{L}) \text { post-operation }(n=779)\end{array}$ & $\begin{array}{l}p- \\
\text { value }\end{array}$ \\
\hline \multicolumn{5}{|l|}{ Cardiac } \\
\hline Chest pain & $21(2.1 \%)$ & * & $17(2.2 \%)$ & $1.000^{+}$ \\
\hline Acute coronary syndrome & * & * & $0(0.0 \%)$ & $0.047^{+}$ \\
\hline Arrhythmia & $25(2.5 \%)$ & $16(7.4 \%)$ & $9(1.2 \%)$ & $\begin{array}{l}< \\
0.001\end{array}$ \\
\hline Pulmonary oedema & $24(2.4 \%)$ & $11(5.1 \%)$ & $13(1.7 \%)$ & 0.004 \\
\hline Cardiorespiratory arrest & * & $0(0.0 \%)$ & * & $1.000^{+}$ \\
\hline \multicolumn{5}{|l|}{ Respiratory } \\
\hline Exacerbation of airways disease & $17(1.7 \%)$ & * & $15(1.9 \%)$ & $0.551^{+}$ \\
\hline Atelectasis & $47(4.7 \%)$ & $12(5.5 \%)$ & $35(4.5 \%)$ & 0.524 \\
\hline Lower respiratory tract infection & $49(4.9 \%)$ & $21(9.7 \%)$ & $28(3.6 \%)$ & $\begin{array}{l}< \\
0.001\end{array}$ \\
\hline Pulmonary embolus & $11(1.1 \%)$ & $8(3.7 \%)$ & $*$ & $\begin{array}{l}<.001^{+} \\
0_{0}\end{array}$ \\
\hline $\begin{array}{l}\text { Requirement for mechanical } \\
\text { ventilation (invasive or non- } \\
\text { invasive) }\end{array}$ & * & $0(0.0 \%)$ & * & $1.000^{+}$ \\
\hline \multicolumn{5}{|l|}{ Gastrointestinal (GI) } \\
\hline Antiemetic given ${ }^{a}$ & $351(35.2 \%)$ & $81(37.3 \%)$ & $270(34.7 \%)$ & 0.467 \\
\hline Constipation $^{\text {b }}$ & $25(2.5 \%)$ & * & $41(5.3 \%)$ & 0.478 \\
\hline Gl bleed & * & * & $0(0.0 \%)$ & $0.218^{+}$ \\
\hline \multicolumn{5}{|l|}{ Neurological } \\
\hline Confusion ${ }^{c}$ & $65(6.5 \%)$ & $22(10.1 \%)$ & $43(5.5 \%)$ & 0.015 \\
\hline Vasovagal episode $^{d}$ & $249(25.0 \%)$ & $54(24.9 \%)$ & $195(25.0 \%)$ & 0.965 \\
\hline Fall & $15(1.5 \%)$ & $5(2.3 \%)$ & $10(1.3 \%)$ & $0.340^{+}$ \\
\hline $\begin{array}{l}\text { Cerebrovascular accident/transient } \\
\text { ischaemic attack }\end{array}$ & * & $0(0.0 \%)$ & * & $1.000^{+}$ \\
\hline \multicolumn{5}{|l|}{ Miscellaneous } \\
\hline Requiring intravenous fluids & $105(10.5 \%)$ & $23(10.6 \%)$ & $82(10.5 \%)$ & 0.975 \\
\hline Hyponatraemia - drop in $\mathrm{Na}<5$ & $157(15.7 \%)$ & $111(51.2 \%)$ & $46(5.9 \%)$ & $\begin{array}{l}<.001 \\
0.001\end{array}$ \\
\hline $\begin{array}{l}\text { Acute kidney injury - Stage } 1,2 \text { or } 3 \\
\text { KDIGO criteria }\end{array}$ & $73(7.3 \%)$ & $31(14.3 \%)$ & $42(5.4 \%)$ & $\begin{array}{l}< \\
0.001\end{array}$ \\
\hline Urinary tract infection & $36(3.6 \%)$ & $10(4.6 \%)$ & $26(3.3 \%)$ & 0.375 \\
\hline Transfusion of blood products & $94(9.4 \%)$ & $45(20.7 \%)$ & $49(6.3 \%)$ & $\begin{array}{l}<.001 \\
0.001\end{array}$ \\
\hline Difficult or delayed mobilisation ${ }^{e}$ & $134(13.5 \%)$ & $38(17.5 \%)$ & $96(12.3 \%)$ & 0.048 \\
\hline Wound-related complications $^{f}$ & $99(9.9 \%)$ & $27(12.4 \%)$ & $72(9.2 \%)$ & 0.164 \\
\hline $\begin{array}{l}\text { Delayed discharge awaiting social } \\
\text { services }\end{array}$ & $87(8.7 \%)$ & $25(11.5 \%)$ & $62(8.0 \%)$ & 0.100 \\
\hline $\begin{array}{l}\text { Transferred to another unit for } \\
\text { acute post-operative care }\end{array}$ & $11(1.1 \%)$ & $5(2.3 \%)$ & $6(0.8 \%)$ & $0.068^{+}$ \\
\hline $\begin{array}{l}\text { Transferred to another unit for } \\
\text { rehabilitation }\end{array}$ & $10(1.0 \%)$ & * & $8(1.0 \%)$ & $1.000^{+}$ \\
\hline
\end{tabular}

All p-values provided by chi-square test apart from ${ }^{+}$which indicates Fisher's Exact Test

* $<5$ patients - number not stated to protect patient confidentiality

${ }^{a}$ Antiemetics may have been given prophylactically as well as to treat symptoms

${ }^{\mathrm{b}}$ Laxative prescription was standard practice during this period. Constipation figures reflect instances where constipation required additional input during the inpatient journey

${ }^{c}$ Confusion includes delirium or confusion related to pre-existing cognitive impairments

${ }^{d}$ Vasovagal includes any instance where light-headedness on standing or mobilising necessitated limitations to mobilisation and/or return to bed. It also includes the less common episode of loss of consciousness

eDifficulty or delayed mobilisation includes people who were slow to mobilise for any reason including pre-existing deficits, knee braces, and/or resolving regional anaesthesia

fWe used a very broad definition for wound-related complications include bleeding requiring change of dressing 
Table 3 Medication use for total group and comparison of those with and without post-operative hyponatraemia

\begin{tabular}{|c|c|c|c|c|}
\hline Medication & $\begin{array}{l}\text { Hyponatraemia }(\mathrm{Na}<135 \mathrm{mmol} / \mathrm{L}) \\
\text { post-operation }(n=217)\end{array}$ & $\begin{array}{l}\text { No hyponatraemia }(\mathrm{Na} \geq 135 \mathrm{mmol} / \\
\text { L) post-operation }(n=783)\end{array}$ & $\begin{array}{l}p- \\
\text { value }^{1}\end{array}$ & $\begin{array}{l}\text { Total group } \\
(n=1000)\end{array}$ \\
\hline Warfarin & $13(6.0 \%)$ & $26(3.3 \%)$ & 0.072 & $39(3.9 \%)$ \\
\hline Other oral anticoagulants & $0(0 \%)$ & $7(0.9 \%)$ & 0.357 & $7(0.7 \%)$ \\
\hline Antiplatelets & $79(36.4 \%)$ & $221(28.2 \%)$ & 0.018 & $300(30 \%)$ \\
\hline Steroids & $7(3.2 \%)$ & $29(3.7 \%)$ & 0.747 & $36(3.6 \%)$ \\
\hline Hypoglycaemic agents & $29(13.4 \%)$ & $54(6.9 \%)$ & 0.002 & $83(8.3 \%)$ \\
\hline Insulin & $7(3.2 \%)$ & $9(1.1 \%)$ & 0.058 & $16(1.6 \%)$ \\
\hline $\mathrm{PPI} / \mathrm{H}_{2}$ receptor antagonist & $105(48.4 \%)$ & $344(43.9 \%)$ & 0.221 & $449(44.9 \%)$ \\
\hline Bisphosphonate & $17(7.8 \%)$ & $68(8.8 \%)$ & 0.704 & $85(8.5 \%)$ \\
\hline Oral calcium & $26(12.0 \%)$ & $69(8.8 \%)$ & 0.153 & $95(9.5 \%)$ \\
\hline Levothyroxine & $36(16.6 \%)$ & $81(10.3 \%)$ & 0.011 & $117(11.7 \%)$ \\
\hline Oral opioid analgesics & $66(30.4 \%)$ & 285 (36.3\%) & 0.111 & $351(35.1 \%)$ \\
\hline Opioid analgesic patch & $18(8.3 \%)$ & $93(11.9 \%)$ & 0.142 & $111(11.1 \%)$ \\
\hline NSAIDs & $68(31.3 \%)$ & $248(31.7 \%)$ & 0.957 & $316(31.6 \%)$ \\
\hline ACEi/ARB & 107 (49.3\%) & $258(33.3 \%)$ & $\begin{array}{l}< \\
0.001\end{array}$ & $365(36.5 \%)$ \\
\hline $\begin{array}{l}\text { Bendrofluazide/indapamide/CCB/ } \\
\text { alpha blocker }\end{array}$ & $99(45.6 \%)$ & $251(32.1 \%)$ & $\begin{array}{l}< \\
0.001\end{array}$ & $350(35.0 \%)$ \\
\hline B-blocker & $58(26.7 \%)$ & $155(19.8 \%)$ & 0.025 & $213(21.3 \%)$ \\
\hline $\begin{array}{l}\text { Antianginals, digoxin and loop and } \\
\text { potassium sparing diuretics }\end{array}$ & $41(18.9 \%)$ & 105 (13.4\%) & 0.040 & $146(14.6 \%)$ \\
\hline Statins & $97(44.7 \%)$ & $294(37.5 \%)$ & 0.130 & $391(39.1 \%)$ \\
\hline Prostate drugs/drugs for incontinence & $16(7.4 \%)$ & $88(11.2 \%)$ & 0.103 & $103(10.3 \%)$ \\
\hline $\begin{array}{l}\text { Hypnotics/antidepressants/ } \\
\text { neuropathic pain/antiepileptics }\end{array}$ & $60(27.6 \%)$ & 259 (33.1\%) & 0.139 & $319(31.9 \%)$ \\
\hline Dementia/PD drugs & $7(3.2 \%)$ & $16(2.0 \%)$ & 0.307 & $23(2.3 \%)$ \\
\hline Inhalers & $28(12.9 \%)$ & $103(13.2 \%)$ & 0.923 & $131(13.1 \%)$ \\
\hline \multicolumn{5}{|l|}{ Antibiotic type } \\
\hline Flucloxacillin & $134(61.8 \%)$ & $565(72.2 \%)$ & 0.003 & $699(69.9 \%)$ \\
\hline Gentamicin & $137(63.1 \%)$ & $568(72.5 \%)$ & 0.007 & $705(70.5 \%)$ \\
\hline Teicoplanin & $80(36.9 \%)$ & $215(27.5 \%)$ & 0.007 & $295(29.5 \%)$ \\
\hline
\end{tabular}

${ }^{1} p$-value comparing those with post-operative hyponatraemia $(\mathrm{Na}<135 \mathrm{mmol} / \mathrm{L})$ and those with normal sodium values $(\mathrm{Na} \geq 135 \mathrm{mmol} / \mathrm{L})$ post-operatively

those maintaining a normal $\mathrm{Na}$ concentration perioperatively.

Sah (2014) found a higher incidence of hyponatraemia $(40 \%)$ in their elective joint arthroplasty population. Similar to our findings, they also found risk factors of post-operative hyponatraemia included pre-operative hyponatremia and older age. They also found female sex, lower body weight, knee in comparison to hip surgery, and bilateral knee arthroplasty to be risk factors. Our finding that age and preoperative $\mathrm{Na}$ concentration independently predicts postoperative hyponatraemia supports the understanding of post-operative hyponatraemia, in this population, as a result of both baseline physiological vulnerability and peri-operative changes. Leung et al. (2012), in their database study, showed that pre-operative hyponatraemia $(\mathrm{Na}<135 \mathrm{mmol} / \mathrm{L})$ predicted longer LOS and increased risk of post-operative complications and 30-day mortality (Leung et al., 2012). Abola et al. (2019) also found that pre-operative hyponatraemia was associated with longer LOS and greater risk of reoperation (Abola et al., 2019). In our study, we found a significantly longer length of stay in those with postoperative hyponatraemia; however, pre- or post-operative sodium levels were not associated with a delayed discharge in multivariate analysis. We also found no significant differences in reattendance at $\mathrm{ED}$, and/or readmission within 90 days between those who had post-operative hyponatraemia and those who did not.

That hyperglycaemia can lower $\mathrm{Na}$ is well-established. Traditional formulae for correcting $\mathrm{Na}$ for hyperglycaemia (Katz, 1973; Hillier et al., 1999) predict a smaller change in sodium than what was observed here, suggesting that there may be other contributory mechanisms. 
Table 4 Logistic regression analysis of factors associated with having post-operative hyponatraemia following elective primary hip and knee arthroplasty

\begin{tabular}{|c|c|c|c|c|}
\hline & $\begin{array}{l}\text { Unadjusted Odds ratio } \\
(95 \% \mathrm{Cl})\end{array}$ & $p$-value & $\begin{array}{l}\text { Adjusted Odds ratio } \\
(95 \% \mathrm{Cl})^{*}\end{array}$ & $p$-value \\
\hline Age (years) & $1.05(1.03-1.07)$ & $<0.001$ & $1.07(1.02-1.13)$ & 0.007 \\
\hline Height (cm) & $0.97(0.95-0.99)$ & 0.001 & $0.95(0.93-1.01)$ & 0.220 \\
\hline Weight (kg) & $0.99(0.98-1.00)$ & 0.019 & $1.02(0.99-1.05)$ & 0.151 \\
\hline \multicolumn{5}{|l|}{ ASA grade } \\
\hline 1 (reference) & 1.0 (reference) & - & 1.0 (reference) & - \\
\hline 2 & $2.09(0.93-4.68)$ & 0.074 & $0.64(0.24-1.78)$ & 0.371 \\
\hline 3 & $2.82(1.20-6.62)$ & 0.017 & $0.51(0.13-1.66)$ & 0.245 \\
\hline Peripheral nerve block & $1.30(0.95-1.78)$ & 0.100 & $1.31(0.56-3.02)$ & 0.534 \\
\hline Pre-operative $\mathrm{Na}$ (mmol/L) & $0.72(0.67-0.77)$ & $<0.001$ & $0.66(0.55,0.78)$ & $<0.001$ \\
\hline Fasting glucose (mmol/L) & $1.38(1.21-1.56)$ & $<0.001$ & $1.33(1.08,1.64)$ & 0.007 \\
\hline Pre-operative haemoglobin (g/L) & $0.98(0.97-0.99)$ & $<0.001$ & $0.98(0.95,1.01)$ & 0.138 \\
\hline \multicolumn{5}{|l|}{ Antibiotic } \\
\hline Flucloxacillin & $0.62(0.45,0.85)$ & 0.003 & $0.50(0.21,1.18)$ & 0.112 \\
\hline Gentamicin $^{+}$ & $0.65(0.47,0.89)$ & 0.007 & & \\
\hline Teicoplanin $^{+}$ & $1.54(1.12-2.12)$ & 0.007 & & \\
\hline \multicolumn{5}{|l|}{ Medication } \\
\hline Warfarin & $1.86(0.94-3.68)$ & 0.076 & $0.55(0.08-3.69)$ & 0.540 \\
\hline Antiplatelets & $1.47(1.07-2.02)$ & 0.018 & $0.65(0.24-1.71)$ & 0.379 \\
\hline Hypoglycaemic agents & $2.09(1.30,3.38)$ & 0.002 & $1.64(0.52,5.16)$ & 0.396 \\
\hline Insulin & $2.88(1.06-7.83)$ & 0.038 & $0.81(0.12-5.64)$ & 0.835 \\
\hline Levothyroxine & $1.73(1.13-2.65)$ & 0.011 & $1.15(0.42-3.17)$ & 0.790 \\
\hline ACEi/ARB & $2.00(1.47,2.71)$ & $<0.001$ & $1.31(0.60,2.86)$ & 0.503 \\
\hline Bendrofluazide/indapamide/ & $1.79(1.32,2.44)$ & $<0.001$ & $0.89(0.41,1.94)$ & 0.769 \\
\hline \multicolumn{5}{|l|}{ CCB/alpha blocker } \\
\hline B-blocker & $1.49(1.05-2.11)$ & 0.026 & $2.54(1.00-6.46)$ & 0.050 \\
\hline Antianginals, digoxin and loop and potassium sparing diuretics & $1.51(1.02-2.25)$ & 0.041 & $0.81(0.31-2.14)$ & 0.676 \\
\hline
\end{tabular}

Logistic regression analysis used to determine factors associated with post-operative hyponatraemia ( $\mathrm{Na}<135 \mathrm{mmol} / \mathrm{L})$

*Adjusted for variables which showed a p-value $<0.10$ in Tables 1 and 2; age, height, weight, ASA grade, peripheral nerve block, pre-operative Na, fasting glucose, pre-operative haemoglobin, flucloxacillin, warfarin, antiplatelets, hypoglycaemic agents, insulin, levothyroxine, ACEi/ARB, bendrofluazide/indapamide/CCB/alpha blocker, B-blocker and antianginals, digoxin and loop and potassium sparing diuretics

${ }^{+}$Gentamicin and teicoplanin not included in multivariate analysis due to multicollinearity. Flucloxacillin kept in multivariate analysis using stepwise backwards approach

Diabetes mellitus has been associated with an increased incidence of hyponatraemia in community studies (Liamis et al., 2013; Mohan et al., 2013) and with increased ADH levels. Hyperglycaemia following arthroplasty has, to date, largely been studied for its association with joint infection (Hwang et al., 2015).

Intra-operative antibiotic use of teicoplanin was associated with a higher incidence of hyponatraemia compared to flucloxacillin and gentamicin. Due to multicollinearity, and using a stepwise backwards approach, flucloxacillin was no longer significantly associated with post-operative hyponatraemia in the multivariate analysis, whilst gentamicin and teicoplanin were excluded from the adjusted model.
Those with post-operative hyponatraemia did have higher rates of inpatient complications which may have contributed to their longer LOS. It must be acknowledged that several of the complications studied (e.g. pulmonary oedema and acute kidney injury) may be reflective of fluid balance issues and are therefore not mutually exclusive to hyponatraemia. Cardiovascular medications were significantly associated with hyponatraemia in univariate analysis, although they lost their significance in the multivariate analysis. Previous findings have reported that cardiovascular medications predict post-operative hyponatraemia (Udy et al., 2005; Hawkins et al., 2003; Mohan et al., 2013; Bissram et al., 2007). The relevant drugs are indicated for both 
Table 5 Serum sodium concentrations for total group, arthroplasty type and with and without post-operative hyponatraemia

\begin{tabular}{|c|c|c|c|c|c|c|c|c|}
\hline & $\begin{array}{l}\text { Hip }(n \\
=493)\end{array}$ & $\begin{array}{l}\text { Knee } \\
(n= \\
425)\end{array}$ & $\begin{array}{l}\text { Uni- } \\
\text { knee } \\
(n= \\
82)\end{array}$ & $\begin{array}{l}p- \\
\text { value }^{1}\end{array}$ & $\begin{array}{l}\text { Hyponatraemia } \\
(\mathrm{Na}<135 \mathrm{mmol} / \mathrm{L}) \\
\text { post-operation }(n \\
=217)\end{array}$ & $\begin{array}{l}\text { No hyponatraemia } \\
(\mathrm{Na} \geq 135 \mathrm{mmol} / \mathrm{L}) \\
\text { post-operation }(n= \\
783)\end{array}$ & $\begin{array}{l}p- \\
\text { value }^{2}\end{array}$ & Total $(n=1000)$ \\
\hline $\begin{array}{l}\text { Post-operative } \mathrm{Na}<135 \mathrm{mmol} / \mathrm{L} \\
(n=1000)\end{array}$ & $\begin{array}{l}100 \\
(20.2 \%)\end{array}$ & $\begin{array}{l}101 \\
(23.8 \%)\end{array}$ & $\begin{array}{l}16 \\
(19.5 \%)\end{array}$ & 0.391 & - & - & - & $217(21.7 \%)$ \\
\hline $\begin{array}{l}\text { Post-operative Na decrease } \geq 5 \\
\mathrm{mmol} / \mathrm{L}(n=1000)\end{array}$ & $\begin{array}{l}* 54 \\
(11.0 \%)\end{array}$ & $\begin{array}{l}{ }^{*} 90 \\
(21.2 \%)\end{array}$ & $\begin{array}{l}13 \\
(15.9 \%)\end{array}$ & $\begin{array}{l}< \\
0.001\end{array}$ & $111(51.1 \%)$ & $106(13.5 \%)$ & $\begin{array}{l}< \\
0.001\end{array}$ & $157(15.7 \%)$ \\
\hline $\begin{array}{l}\text { Pre-assessment } \mathrm{Na}(\mathrm{mmol} / \mathrm{L})(n \\
=999)\end{array}$ & $\begin{array}{l}139.0 \\
(138.0- \\
141.0)\end{array}$ & $\begin{array}{l}140.0 \\
(138.0- \\
141.0)\end{array}$ & $\begin{array}{l}140.0 \\
(138.0- \\
141.0)\end{array}$ & 0.129 & $138.0(136.0-140.0)$ & $140.0(138.0-141.0)$ & $\begin{array}{l}< \\
0.001\end{array}$ & $139.0(138.0-141.0)$ \\
\hline $\begin{array}{l}\text { Pre-operative } \mathrm{Na}(\mathrm{mmol} / \mathrm{L})(n= \\
723)\end{array}$ & $\begin{array}{l}139.0 \\
(138.0- \\
141.0)\end{array}$ & $\begin{array}{l}139.0 \\
(138.0- \\
141.0)\end{array}$ & $\begin{array}{l}140.0 \\
(138.0- \\
141.0)\end{array}$ & 0.807 & $138.0(136.0-140.0)$ & $140.0(138.0-141.0)$ & $\begin{array}{l}< \\
0.001\end{array}$ & $139.0(138.0-141.0)$ \\
\hline Day $1 \mathrm{Na}(\mathrm{mmol} / \mathrm{L})(n=994)$ & $\begin{array}{l}138.0 \\
(136.0- \\
139.0)\end{array}$ & $\begin{array}{l}138.0 \\
(136.0- \\
139.0)\end{array}$ & $\begin{array}{l}138.0 \\
(136.0- \\
139.0)\end{array}$ & 0.794 & $134.0(133.0-136.0)$ & $138.0(137.0-139.0)$ & $\begin{array}{l}< \\
0.001\end{array}$ & $138.0(136.0-139.0)$ \\
\hline Day $2 \mathrm{Na}(\mathrm{mmol} / \mathrm{L})(n=501)$ & $\begin{array}{l}* 137.0 \\
(135.0- \\
139.0)\end{array}$ & $\begin{array}{l}* 137.0 \\
(134.0- \\
139.0)\end{array}$ & $\begin{array}{l}137.0 \\
(135.0- \\
140.0)\end{array}$ & 0.039 & $134.0(132.0-135.0)$ & $138.0(137.0-140.0)$ & $\begin{array}{l}< \\
0.001\end{array}$ & $137.0(135.0-139.0)$ \\
\hline $\begin{array}{l}\text { Minimum post-operative } \mathrm{Na} \\
(\mathrm{mmol} / \mathrm{L})(n=1000)\end{array}$ & $\begin{array}{l}137.0 \\
(135.0- \\
138.0)\end{array}$ & $\begin{array}{l}137.0 \\
(135.0- \\
138.0)\end{array}$ & $\begin{array}{l}137.0 \\
(135.0- \\
138.0)\end{array}$ & 0.475 & $133.0(131.0-134.0)$ & $138.0(136.0-139.0)$ & $\begin{array}{l}< \\
0.001\end{array}$ & $137.0(135-138.0)$ \\
\hline $\begin{array}{l}\text { Na prior to discharge }(\mathrm{mmol} / \mathrm{L}) \\
(n=988)\end{array}$ & $\begin{array}{l}138.0 \\
(136.0- \\
139.0)\end{array}$ & $\begin{array}{l}137.0 \\
(135.0- \\
139.0)\end{array}$ & $\begin{array}{l}138.0 \\
(136.0- \\
139.0)\end{array}$ & 0.332 & $134.0(133.0-137.0)$ & $138.0(137.0-139.0)$ & $\begin{array}{l}< \\
0.001\end{array}$ & $138.0(136.0-139.0)$ \\
\hline Change in $\mathrm{Na}(\mathrm{mmol} / \mathrm{L})(n=999)$ & $\begin{array}{l}*-2.0 \\
(-4.0 \text { to } \\
-1.0)\end{array}$ & $\begin{array}{l}*-3.0 \\
(-5.0 \\
\text { to }- \\
1.0)\end{array}$ & $\begin{array}{c}-3.0 \\
(- \\
4.0 \\
\text { to - } \\
1.0)\end{array}$ & 0.012 & $-6.0(-8.0$ to -4.0$)$ & $-2.0(-3.0$ to -1.0$)$ & $\begin{array}{l}< \\
0.001\end{array}$ & $-3.0(-4.0$ to -1.0$)$ \\
\hline Blood transfusion & $\begin{array}{l}63 \\
(12.4 \%)\end{array}$ & $\begin{array}{l}31 \\
(7.3 \%)\end{array}$ & $\begin{array}{l}0 \\
(0.0 \%)\end{array}$ & $\begin{array}{l}< \\
0.001\end{array}$ & $44(20.3 \%)$ & $50(6.4 \%)$ & $\begin{array}{l}< \\
0.001\end{array}$ & $94(9.4 \%)$ \\
\hline 24-h blood loss $(n=989)$ & $\begin{array}{l}* 1252.4 \\
(972.6- \\
1585.0)\end{array}$ & $\begin{array}{l}* 1064.5 \\
(809.7- \\
1320.8)\end{array}$ & $\begin{array}{l}* 535.2 \\
(308.1- \\
763.6)\end{array}$ & $\begin{array}{l}< \\
0.001\end{array}$ & $\begin{array}{l}1133.7(869.1- \\
1515.2)\end{array}$ & $1109.0(798.5-1402.4)$ & 0.128 & $\begin{array}{l}1113.7(810.8- \\
1428.1)\end{array}$ \\
\hline 48-h blood loss ( $n=989$ ) & $\begin{array}{l}{ }^{*} 1405.2 \\
(1017.8- \\
1794.8)\end{array}$ & $\begin{array}{l}{ }^{*} 1264.7 \\
(929.9- \\
1567.1)\end{array}$ & $\begin{array}{l}* 646.2 \\
(357.5- \\
808.9)\end{array}$ & $<0.001$ & $\begin{array}{l}1390.3(989.0- \\
1817.9)\end{array}$ & $1264.8(885.0-1633.9)$ & 0.003 & $\begin{array}{l}1287.7(899.9- \\
1675.8)\end{array}$ \\
\hline
\end{tabular}

Median (IQR) used for continuous variables and $\mathrm{n}$ (\%) used for categorical variables

Kruskal-Wallis test used to compare continuous variables between arthroplasty type and Mann-Whitney $\mathrm{U}$ test used to compare continuous variables between those with and without post-operative hyponatraemia

*Groups which show significant difference

${ }^{1} p$-value comparing those who underwent total hip arthroplasty, total knee arthroplasty and uni-compartmental knee arthroplasty

${ }^{2} p$-value comparing those with post-operative hyponatraemia ( $\mathrm{Na}<135 \mathrm{mmol} / \mathrm{L}$ ) and those with normal sodium values (Na $\left.\geq 135 \mathrm{mmol} / \mathrm{L}\right)$ post-operatively

hypertension and heart failure. It is not clear whether these associations between drugs and postoperative hyponatraemia are due to the drugs themselves, or are by virtue of underlying comorbidities predisposing to hyponatraemia. A propensity for heart failure and fluid retention could exacerbate the appropriate peri-operative release of $\mathrm{ADH}$ that is likely the main driver of post-operative hyponatraemia. Of note, our practice changed during the time of this audit and instead of being judged on a caseby-case basis, a blanket withholding of angiotensin converting enzyme inhibitors and angiotensin receptor blockers, in particular over the peri-operative period, was introduced. We found decreased height and decreased weight in those with post-operative hyponatraemia, but no significant difference in BMI between those with post-operative hyponatraemia and those with normal Na. Sah (2014) also reported lower body weight in those with post-operative hyponatraemia. It is possible that those with lower height and weight were subject to a relative excess of intravenous fluids infusion post-operatively. Whilst we have included infused fluid volumes (on day of surgery, available for $n=392$ only) in our analyses (Table 1), we did not have net fluid balance available for sufficient patients to do this. 


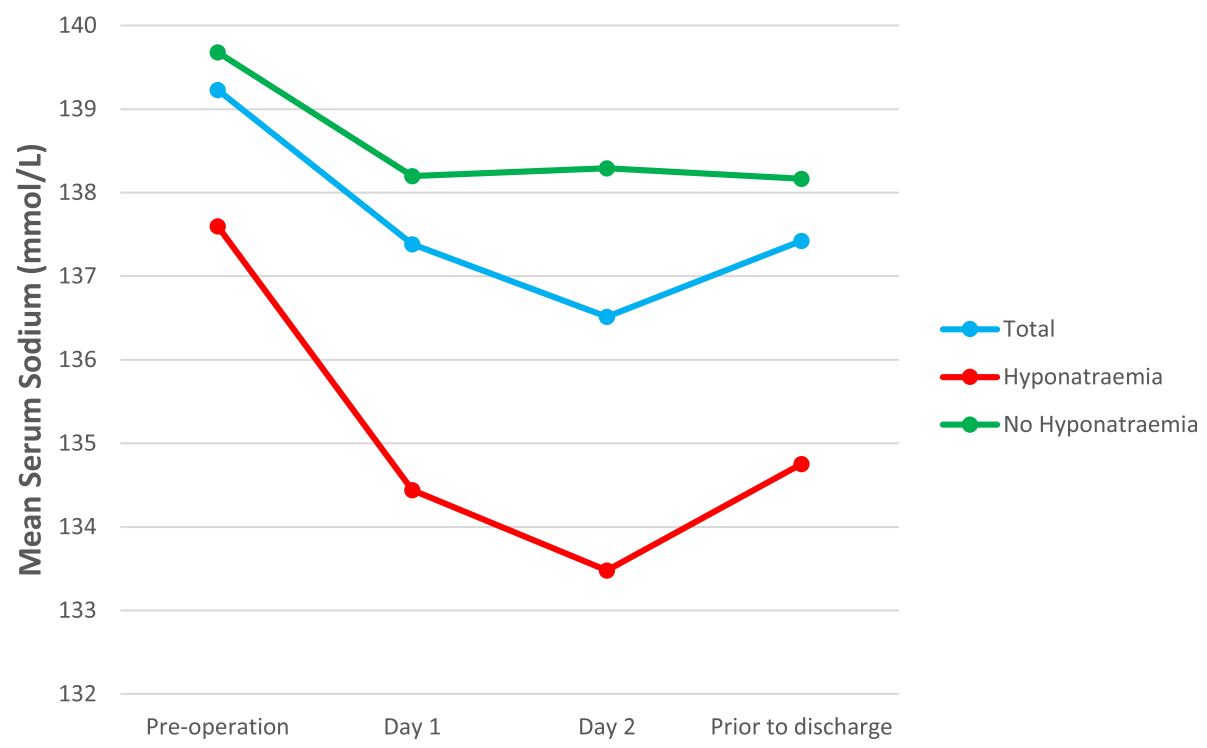

Fig. 1 Mean serum sodium for total group and comparison of those with and without post-operative hyponatraemia

It is noteworthy that there was no significant increase in reattendance at ED and/or readmission to hospital for those patients who developed post-operative hyponatraemia. Our rates of discharge with hyponatraemia (10.8\%) were lower than those reported in other series, with Hennrikus et al. (2015) reporting $28 \%$ of patients to still be hyponatraemic on discharge from hospital (Hennrikus et al., 2015). Hennrikus et al. (2015) included not only THA and TKA but also spine fusion and other surgeries, such as shoulder arthroplasty and foot and ankle procedures. Our findings, therefore, challenge the rationale for keeping otherwise well patients in hospital solely to monitor $\mathrm{Na}$. It has been suggested that the standard reference range for $\mathrm{Na}$ utilised by most laboratories may not be universally applicable to inpatients (McKee et al., 2016). Our data would support this concept and the notion that milder degrees of hyponatraemia are common and probably do not require extensive investigation or treatment. We suggest that otherwise well patients with mild hyponatraemia can safely be discharged and

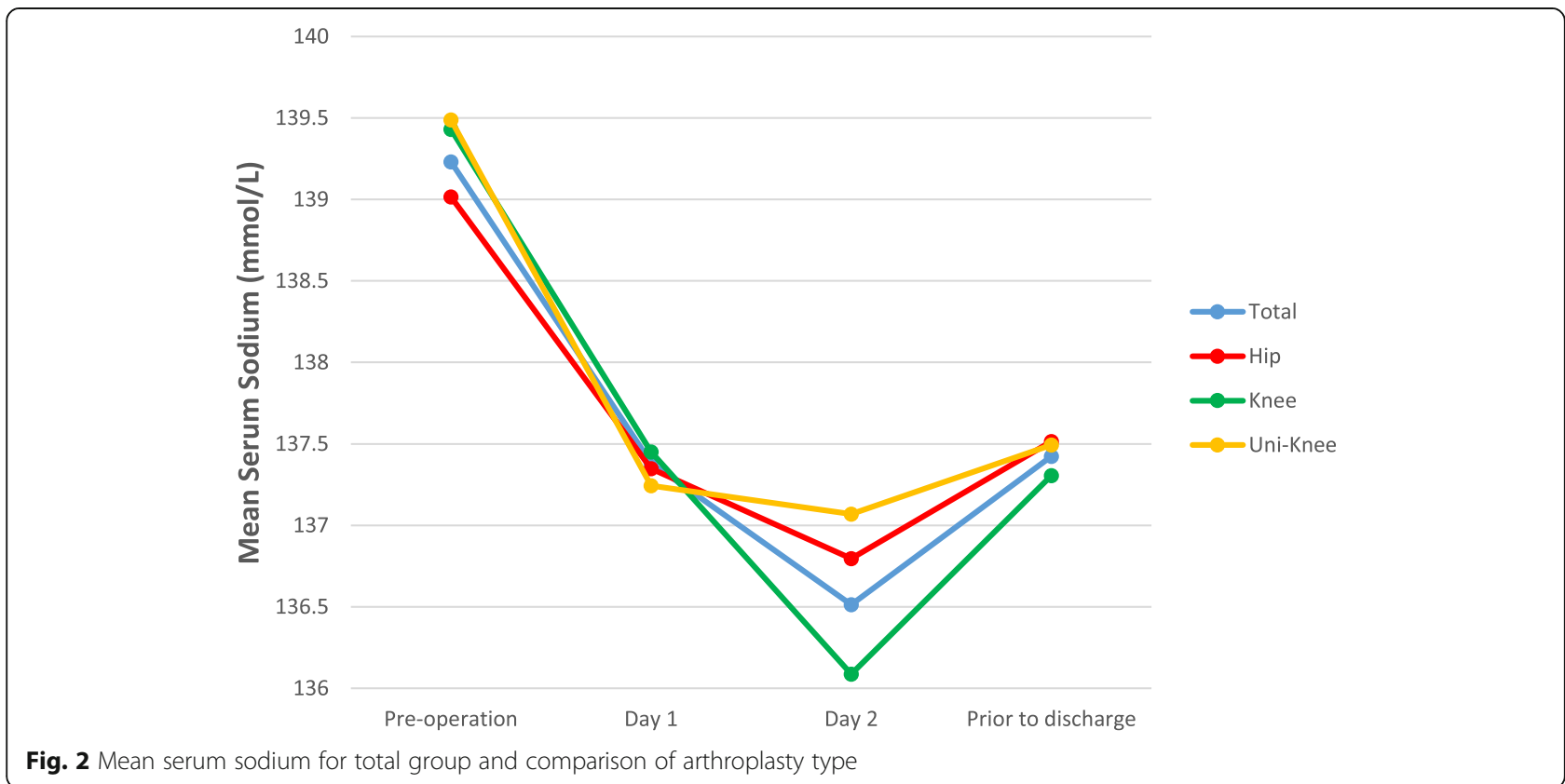




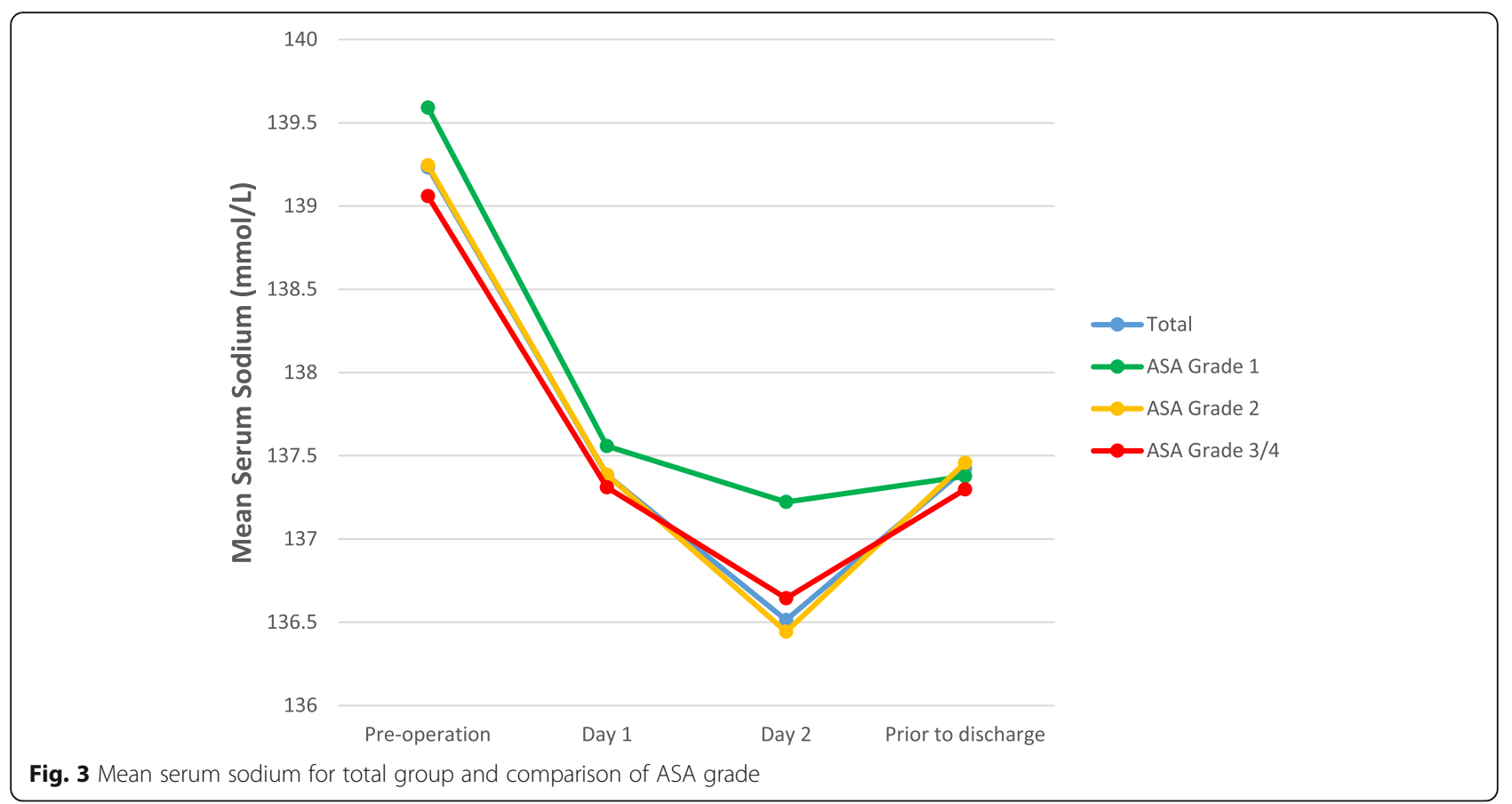

followed up in the community. It is important to note that this is an observational finding. Evidence directly comparing inpatient and outpatient management of post-operative hyponatraemia is required.

The association between post-operative hyponatraemia and confusion did not withstand multivariate analysis, suggesting other factors such as age, pre-operative $\mathrm{Na}$, ASA grade, type of surgery, and transfusion may have been mediating the relationship. It should be noted that we were not able to further categorise confusion, which may have been due to delirium, or pre-existing cognitive impairment. It is also likely, given other estimates from our population (Cunningham et al., 2017), that the incidence of confusion, due to either cause, was underestimated in this audit. There were no significant differences in falls or vasovagal episodes between those with and those without hyponatraemia. This is in contrast to the findings of a systematic review by Corona et al. (2018), which reported hyponatraemia to be significantly associated with an increased risk of falls across all 15 included studies (Corona et al., 2018). Ahamed et al. (2014) also found hyponatraemia to be independently associated with increased risk of admission-associated falls in their case-control study of medical inpatients (Ahamed et al., 2014).

The lack of effect of duration of surgery or blood loss during surgery on post-operative hyponatraemia in our cohort is contrary to other reports (Hennrikus et al., 2015). Our values of both are low (150 ml of blood loss in those with and those without hyponatraemia, and 53 min duration of surgery for those with hyponatraemia vs
$55 \mathrm{~min}$ for those without hyponatraemia), suggesting we may be below the threshold where it makes a difference. For example, Hennrikus et al. (2015) noted significant differences in both duration of surgery and blood loss; duration of surgery was $2.6 \mathrm{~h}$ in those with no hyponatraemia vs $2.9 \mathrm{~h}$ in those who developed hyponatraemia post-operatively, and blood loss was $261 \mathrm{ml}$ in those with no hyponatraemia vs $332 \mathrm{ml}$ in those who developed hyponatraemia post-operatively (Hennrikus et al., 2015). Gender differences have previously been described in some studies, with females at a higher risk of hyponatraemia than males (Mohan et al., 2013), whilst other studies have found no gender differences (Hawkins et al., 2003), as in this audit.

\section{Strengths}

Strengths of this study include the high numbers of participants with clinical chart-level information, including preoperative drug information. The patients included in this audit are representative of the UK arthroplasty population with a similar mean age, gender ratio, and ASA grading to the National Joint Registry (NJR) population captured in the 10th Annual NJR Report 2013 for England, Wales and Northern Ireland (NJR, 2013) (Supplementary Table 4). A wealth of patient level data not included in other studies, or captured by the electronic systems on which larger studies are based, are presented here.

\section{Limitations}

Available pre-operative $\mathrm{Na}$ measurements were taken in the $24 \mathrm{~h}$ prior to surgery for 723 patients, but for 276 
patients, pre-operative $\mathrm{Na}$ was taken from the preoperative assessment which varied between 1 and 552 days before surgery. The last recorded $\mathrm{Na}$ prior to discharge may have been taken more than $24 \mathrm{~h}$ prior to discharge. Variables differed in the extent of missing data, with some variables having more missing data than others.

Serum and urinary osmolarities, treatment of postoperative hyponatraemia, for example, fluid restriction, administered intravenous fluid volumes and daily fluid balance, and whether cardiac drugs were held over the peri-operative period have not been described. A validated comorbidity score has not been used preoperatively, although ASA grade and medications can give some indication of pre-operative comorbidity. Information regarding complications was recorded pragmatically from the notes and strict criteria were not used. This was a single-surgeon, single-centre series which could influence generalisability.

\section{Conclusion}

In this audit of 1000 THA, TKA, and UKA patients, 217 (21.7\%) had post-operative hyponatraemia. Postoperative hyponatraemia can, to an extent, be predicted. We found no evidence that those discharged with hyponatraemia have more reattendance at ED or readmission to hospital. As we move towards better planned and more efficient inpatient stays for arthroplasty patients, comprehensive pre-operative risk assessment is necessary and must take age, pre-operative $\mathrm{Na}$, and medications into account. Post-operative monitoring and management of post-operative hyponatraemia must be tailored to the individual. We propose that mild ( $\mathrm{Na}$ 130-134 mmol/L) hyponatraemia may be of little to no consequence in the immediate post-operative period and suggest that further investigation would be valuable to determine if moderate ( $\mathrm{Na} 125-129 \mathrm{mmol} / \mathrm{L}$ ) hyponatraemia in otherwise well individuals can be managed in the community. Evidence directly comparing management strategies would be preferable.

\section{Abbreviations}

ADH: Antidiuretic hormone; LOS: Length of stay; SIADH: Syndrome of inappropriate antidiuretic hormone secretion; UK: United Kingdom; THA: Total hip arthroplasty; TKA: Total knee arthroplasty; UKA: Unicompartmental knee arthroplasty; LCS: Low contact stress; MRSA: Methicillinresistant Staphylococcus aureus; GAIN: Guidelines and Audit Implementation Network

\section{Supplementary Information}

The online version contains supplementary material available at https://doi. org/10.1186/s13741-021-00197-1

Additional file 1: Supplementary Table 1. Logistic regression analysis of factors associated with having confusion following elective primary hip and knee arthroplasty. Supplementary Table $\mathbf{2}$. Logistic regression analysis of factors associated with having a delayed discharge (length of stay over 3 days) following elective primary hip and knee arthroplasty. Supplementary Table 3. Reasons for reattendance/readmission in those with post-operative hyponatraemia. Supplementary Table 4. Comparison of demographics of hip and knee replacements patients in the current audit of 1,000 Joints and the 10th Annual National Joint Registry Report 2013

Acknowledgements

We are grateful to Professor lan Young who reviewed earlier versions of the manuscript.

\section{Authors' contributions}

EC completed the data collection and wrote the manuscript, NG completed the data analysis, $\mathrm{PH}$ contributed to the writing of the manuscript, LB completed the data collection, and DB conceptualised the research. The authors read and approved the final manuscript.

Funding

This study has been supported by the Trauma and Orthopaedics Research Charity (https://torcni.org/).

\section{Availability of data and materials}

The datasets used and/or analysed during the current study are available from the corresponding author on reasonable request.

\section{Declarations}

Ethics approval and consent to participate

Not applicable

Consent for publication

Not applicable

\section{Competing interests}

The authors declare that they have no competing interests.

\section{Author details}

${ }^{1}$ Centre for Public Health (Queen's University, Belfast), Institute of Clinical Sciences, Royal Victoria Hospital, Belfast BT12 6BA, Northern Ireland.

${ }^{2}$ Outcomes Unit, Musgrave Park Hospital, Belfast Health and Social Care Trust, Stockman's Lane, Belfast BT9 7JB, Northern Ireland. ${ }^{3}$ Centre for Medical Education, Queen's University Belfast, Mulhouse Road, Belfast BT12 6BA, Northern Ireland. ${ }^{4}$ Department of Clinical Biochemistry, Royal Victoria Hospital, Belfast BT12 6BA, Northern Ireland.

Received: 1 December 2020 Accepted: 31 May 2021

Published online: 03 August 2021

\section{References}

Abola MV, Tanenbaum JE, Bomberger TT, Knapik DM, Fitzgerald SJ, Wera GD. Preoperative hyponatremia is associated with reoperation and prolonged length of hospital stay following total knee arthroplasty. J Knee Surg. 2019; 32(4):344-51. https://doi.org/10.1055/s-0038-1641156.

Ahamed S, Anpalahan M, Savvas S, Gibson S, Torres J, Janus E. Hyponatraemia in older medical patients: implications for falls and adverse outcomes of hospitalisation. Intern Med J. 2014:44(10):991-7. https://doi.org/10.1111/imj.12535.

Bissram M, Scott FD, Liu L, Rosner MH. Risk factors for symptomatic hyponatraemia: the role of pre-existing asymptomatic hyponatraemia. Intern Med J. 2007;37(3):149-55. https://doi.org/10.1111/j.1445-5994.2006.01294.x.

Biswas M, Davies JS. Hyponatraemia in clinical practice. Postgrad Med J. 2007; 83(980):373-8. https://doi.org/10.1136/pgmj.2006.056515.

Cecconi M, Hochrieser H, Chew M, Grocott M, Hoeft A, Hoste A, et al. Preoperative abnormalities in serum sodium concentrations are associated with higher in-hospital mortality in patients undergoing major surgery. $\mathrm{Br} \mathrm{J}$ Anaesth. 2016;116(1):63-9. https://doi.org/10.1093/bja/aev373.

Corona G, Norello D, Parenti G, Sforza A, Maggi M, Peri A. Hyponatremia, falls and bone fractures: a systematic review and meta-analysis. Clin Endocrinol (Oxf). 2018;89(4):505-13. https://doi.org/10.1111/cen.13790. 
Cunningham EL, Mawhinney T, Beverland D, O'Brien S, McAuley DF, Cairns R, et al. Observational cohort study examining apolipoprotein E status and preoperative neuropsychological performance as predictors of post-operative delirium in an older elective arthroplasty population. Age Ageing. 2017;46(5): 779-86. https://doi.org/10.1093/ageing/afx042.

GAIN (2010) Hyponatraemia in adults (on or after 16th birthday). Guidelines and audit implementation network. https://www.rqia.org.uk/RQIA/files/9f/9f2 9d996-722a-4aff-8937-59b937602070.pdf (Accessed 20th Nov 2020).

Guglielminotti J, Tao S, Maury E, Fierobe L, Mantz J, Desmonts JM. Hyponatremia after hip arthroplasty may be related to a translocational rather than to a dilutional mechanism. Crit Care Med. 2003;31(2):442-8. https://doi.org/10.1 097/01.CCM.0000045547.12683.63.

Harris B, Schopflin C, Khaghani C, Edwards M, collaborators from the Southcoast Perioperative Audit and Research Collaboration (SPARC). Perioperative intravenous fluid prescribing: a multi-centre audit. Perioper Med (Lond). 2015; 8(4):15.

Hawkins RC. Age and gender as risk factors for hyponatremia and hypernatremia. Clin Chim Acta. 2003;337(1-2):169-72. https://doi.org/10.1016/j.cccn.2003.08.001.

Hennrikus E, Ou G, Kinney B, Lehman E, Grunfeld R, Wieler J, et al. Prevalence, timing, causes, and outcomes of hyponatremia in hospitalized orthopaedic surgery patients. J Bone Joint Surg Am. 2015;97(22):1824-32. https://doi.org/1 0.2106/JBJS.0.00103.

Hillier TA, Abbott RD, Barrett EJ. Hyponatremia: evaluating the correction factor for hyperglycemia. Am J Med. 1999;106(4):399-403. https://doi.org/10.1016/ S0002-9343(99)00055-8

Hwang JS, Kim SJ, Bamne AB, Na YG, Kim TK. Do glycemic markers predict occurrence of complications after total knee arthroplasty in patients with diabetes? Clin Orthop Relat Res. 2015;473(5):1726-31. https://doi.org/10.1007/ s11999-014-4056-1

Katz MA. Hyperglycemia-induced hyponatremia-calculation of expected serum sodium depression. N Engl J Med. 1973;289(16):843-4. https://doi.org/10.1 056/NEJM197310182891607.

Kildow BJ, Karas V, Howell E, Green CL, Baumgartner WT, Penrose CT, et al. The utility of basic metabolic panel tests after total joint arthroplasty. J Arthroplasty. 2018;33(9):2752-8. https://doi.org/10.1016/j.arth.2018.05.003.

Leung AA, McAlister FA, Rogers SO, Pazo V, Wright A, Bates DW. Preoperative hyponatremia and perioperative complications. Arch Intern Med. 2012; 172(19):1474-81. https://doi.org/10.1001/archinternmed.2012.3992.

Liamis G, Rodenburg EM, Hofman A, Zietse R, Stricker BH, Hoorn EJ. Electrolyte disorders in community subjects: prevalence and risk factors. Am J Med. 2013;126(3):256-63. https://doi.org/10.1016/j.amjmed.2012.06.037.

Madsen CM, Jantzen C, Lauritzen JB, Abrahamsen B, Jorgensen HL. Hyponatremia and hypernatremia are associated with increased 30-day mortality in hip fracture patients. Osteoporos Int. 2016;27(1):397-404. https://doi.org/10.1007/ s00198-015-3423-4.

McCausland FR, Wright J, Waikar SS. Association of serum sodium with morbidity and mortality in hospitalized patients undergoing major orthopedic surgery. J Hosp Med. 2014;9(5):297-302. https://doi.org/10.1002/jhm.2168.

McKee M, Exall S, Stuckler D, Wolff A. (2016) 'Normal' serum sodium concentration among inpatients over 65 admitted to hospital: an observational study. Postgrad Med J. 2016;92(1083):21-6. https://doi.org/1 0.1136/postgradmedj-2015-133482

Mohan S, Gu S, Parikh A, Radhakrishnan J. Prevalence of hyponatremia and association with mortality: results from NHANES. Am J Med. 2013;126(12): 1127-37.e1.

Nagler EV, Vanmassenhove J, van der Veer SN, Nistor I, Van Biesen W, Webster $A C$, et al. Diagnosis and treatment of hyponatremia: a systematic review of clinical practice guidelines and consensus statements. BMC Med. 2014;12(1): 231. https://doi.org/10.1186/s12916-014-0231-1.

National Joint Registry (NJR). National Joint Registry for England, Wales and Northern Ireland: 10th Annual report. 2013. [Online] Available at: http://www. njrcentre.org.uk/njrcentre/portals/0/documents/england/reports/10th_annua I_report/njr\%2010th\%20annual\%20report\%202013\%20b.pdf (Accessed 27th Nov 2020).

National Joint Registry (NJR). National Joint Registry for England, Wales, Northern Ireland and the Isle of Man: 15th Annual Report. 2018. [Online] Available at: https://reports.njrcentre.org.uk/Portals/0/PDFdownloads/NJR\%2015th\%20A nnual\%20Report\%202018.pdf (Accessed $27^{\text {th }}$ Nov 2020).
Ogonda L, Hill J, Doran E, Dennison J, Stevenson M, Beverland D. Aspirin for thromboprophylaxis after primary lower limb arthroplasty: early thromboembolic events and 90 day mortality in 11,459 patients. Bone Joint J. 2016;98-B(3):341-8. https://doi.org/10.1302/0301-620X.98B3.36511.

Park SJ, Shin JI. Inflammation and hyponatremia: an underrecognized condition? Korean J Pediatr. 2013;56(12):519-22. https://doi.org/10.3345/ kjp.2013.56.12.519.

Rondon H, Badireddy M. Hyponatremia. StatPearls [Internet]. Treasure Island (FL): StatPearls Publishing; 2021.

Rudge JE, Kim D. New-onset hyponatraemia after surgery for traumatic hip fracture. Age Ageing. 2014;43(6):821-6.

Sah AP. Hyponatremia after primary hip and knee arthroplasty: incidence and associated risk factors. Am J Orthop (Belle Mead NJ). 2014;43(4):E69-73.

Spasovski G, Vanholder R, Allolio B, Annane D, Ball S, Bichet D, et al. Hyponatraemia Guideline Development Group. Clinical practice guideline on diagnosis and treatment of hyponatraemia. Nephrol Dial Transplant. 2014; 29(Suppl 2):1-39.

Tinning CG, Cochrane LA, Singer BR. Analysis of hyponatraemia associated postoperative mortality in 3897 hip fracture patients. Injury. 2015;46(7):1328-32. https://doi.org/10.1016/j.injury.2015.03.035.

Udy A, Deacy N, Barnes D, Sigston P. Tramadol-induced hyponatraemia following unicompartmental knee replacement surgery. Anaesthesia. 2005;60(8):814-6. https://doi.org/10.1111/j.1365-2044.2005.04260.x.

Upadhyay A, Jaber BL, Madias NE. Incidence and prevalence of hyponatremia. Am J Med. 2006;119(7A):S30-5. https://doi.org/10.1016/j.amjmed.2006.05.005.

Verbalis JG, Goldsmith SR, Greenberg A, Korzelius C, Schrier RW, Sterns RH, et al. Diagnosis, evaluation, and treatment of hyponatremia: expert panel recommendations. Am J Med. 2013;126(10 Suppl 1):S1-42. https://doi.org/1 0.1016/j.amjmed.2013.07.006.

\section{Publisher's Note}

Springer Nature remains neutral with regard to jurisdictional claims in published maps and institutional affiliations.
Ready to submit your research? Choose BMC and benefit from:

- fast, convenient online submission

- thorough peer review by experienced researchers in your field

- rapid publication on acceptance

- support for research data, including large and complex data types

- gold Open Access which fosters wider collaboration and increased citations

- maximum visibility for your research: over $100 \mathrm{M}$ website views per year

At BMC, research is always in progress.

Learn more biomedcentral.com/submissions 